University of Nebraska - Lincoln

DigitalCommons@University of Nebraska - Lincoln

Paul Burrow Publications

Research Papers in Physics and Astronomy

December 1987

\title{
Magnetically Induced Circular Polarization
}

\author{
K.L. Stricklett \\ University of Nebraska - Lincoln \\ D.J. Burns \\ University of Nebraska - Lincoln, djburns@uneb.edu \\ Paul Burrow \\ University of Nebraska - Lincoln, pburrow1@unl.edu
}

Follow this and additional works at: https://digitalcommons.unl.edu/physicsburrow

Part of the Physics Commons

Stricklett, K.L.; Burns, D.J.; and Burrow, Paul, "Magnetically Induced Circular Polarization" (1987). Paul Burrow Publications. 21.

https://digitalcommons.unl.edu/physicsburrow/21

This Article is brought to you for free and open access by the Research Papers in Physics and Astronomy at DigitalCommons@University of Nebraska - Lincoln. It has been accepted for inclusion in Paul Burrow Publications by an authorized administrator of DigitalCommons@University of Nebraska - Lincoln. 


\title{
Magnetically induced circular polarization
}

\author{
K. L. Stricklett, D. J. Burns, and P. D. Burrow \\ Department of Physics and Astronomy, University of Nebraska-Lincoln, Lincoln, Nebraska 68588-0111
}

(Received 27 May 1987)

\begin{abstract}
Helium atoms excited to an aligned $(1 s 3 d)^{3} D$ state are produced by electron impact parallel to a magnetic field. The initial alignment of the atomic state evolves into an orientation with finite angular momentum along the magnetic field direction and thus gives rise to a circularly polarized component in the $(1 s 3 d)^{3} D \rightarrow(1 s 2 p)^{3} P$ radiation propagating parallel to the magnetic field. Circular-polarization fractions of a few percent are observed, in general agreement with theory. The measured polarization fraction is found to be pressure dependent, indicating that secondary excitation and cascade processes must be included for a complete description.
\end{abstract}

\section{INTRODUCTION}

This work reports an experimental demonstration of the mechanism postulated by Kemp, Macek, and Nehring $^{1}$ for the creation of orientation from an initially aligned system in an external magnetic field. In the context of the present paper, "alignment" and "orientation" characterize asymmetries in the populations of the various magnetic sublevels of an excited atomic state. By choice of the appropriate experimental geometry, only alignment is created by the impact excitation process. The presence of circularly polarized light from the decaying state indicates a nonzero angular momentum, that is, an orientation along the magnetic field direction. Such orientation can only arise during the evolution of an initially aligned state following excitation. The mechanism for this process is the subject of the work by Kemp et al. ${ }^{1}$

An external magnetic field acting on an isolated state will only induce a precession of the angular momentum vector about the field direction. However, if fine structure is not experimentally resolved, the combined effects of the magnetic field interaction with the orbital and spin magnetic moments and the spin-orbit interaction may conspire to alter the relative populations of the magnetic sublevels. ${ }^{2}$ The effects of these interactions may, in principle, be observed in the rapid time development of the alignment as in quantum-beat experiments. Additionally, Kemp et al., ${ }^{1}$ using a semiclassical argument, show that the time developments of $L_{z}$ parallel and antiparallel to the magnetic field are not equivalent for an initially aligned system. They show further that this asymmetry results in a time-averaged shift in the populations of the magnetic sublevels and thus the creation of orientation, manifested by the production of a circularly polarized component in the emitted light. General discussions of the theoretical treatment of this problem may be found in Blum, ${ }^{3}$ Fano and Macek, ${ }^{4}$ and Lombardi. ${ }^{5}$

There has not been a definitive demonstration of this effect. Kemp et al. describe "cursory" evidence, in which circular polarization is observed in the light emit- ted from gas discharge tubes placed in a magnetic field. However, the nature of their experiment makes these results difficult to interpret. Other closely related effects have been observed; the Hanle effect ${ }^{6}$ involves rotation of alignment in a magnetic field. Additionally, Lehmann ${ }^{7}$ has shown a magnetic-field-strength dependence for nuclear orientation in optical pumping experiments.

\section{EXPERIMENTAL PROCEDURE}

Full quantum-mechanical calculations ${ }^{2}$ of magnetically induced conversion of alignment to orientation have been carried out for several excited states of helium. In the present study, we have chosen to examine the $3{ }^{3} \mathrm{D}$ state of helium to permit comparison with these results from theory. The excited atoms are created, with alignment, by electron-impact excitation in a configuration in which the initial electron momentum is parallel to a magnetic field of variable strength. The $3{ }^{3} D \rightarrow 2{ }^{3} P$ radiation at $5876 \AA$ is viewed "end-on," that is, parallel to the magnetic field direction, and the circularpolarization fraction is measured.

In our experiment, the scattered electron is not detected; hence, the excitation geometry has axial symmetry, and only the $A_{0}$ and $O_{0}$ elements of the full alignment and orientation tensors may be nonzero. ${ }^{3,4}$ It is useful to note here that this leads to a simple interpretation of alignment and orientation. ${ }^{5}$ An aligned system has states of different $\left|m_{l}\right|$ populated unequally, while the populations of the $m_{l}$ and $-m_{l}$ states are equal. An oriented system has differing populations for states of $m_{l}$ and $-m_{l}$. Assuming that the excitation process is rapid on the time scale of the Zeeman and spin-orbit precessions, that is, that the sudden-impact approximation is valid, the magnetic field may be neglected during excitation, and the excitation process is independent of the direction of the initial electron momentum. Thus, for the geometry of this experiment, the alignments created by electron impact parallel and antiparallel to the magnetic field direction are identical. This additional symmetry requires the populations of the $m_{l}$ and $-m_{l}$ states to be equal, ${ }^{3}$ that is, net angular momentum may not be 
transferred to the ensemble of atoms in this excitation geometry, and the initial orientation must be zero.

The electron-beam apparatus used to generate the excited atoms is shown schematically in Fig. 1. Its configuration is essentially that of an electron transmission spectrometer as developed by Sanche and Schulz. ${ }^{8}$ Several features of this apparatus are advantageous for the present study. Firstly, a magnetic field parallel to the motion of the electrons in the collision region is employed for beam collimation. The operation of the device is insensitive to the magnetic field strength which, in the present work, was varied over the range from 90-350 G. Secondly, the configuration of the electronbeam source is that of a trochoidal monochromator, ${ }^{9}$ which features a cathode displaced from the electronbeam path through the collision region which is the optical axis of the apparatus. This simplifies the shielding of stray light from the cathode and heater.

The operation of the trochoidal monochromator is described more completely in the literature. ${ }^{9-12}$ Here we note briefly that the electrons are injected into a region in which an electric field is applied perpendicular to the guiding magnetic field. The electrons thus acquire a transverse drift velocity, $\mathrm{V}_{d}=\mathrm{E} \times \mathrm{B} / \mathrm{B}^{2}$, and are dispersed according to the time of passage through the crossed-field region. A portion of the electron distribution is selected at the defining aperture which lies on the optical axis of the apparatus. Normally, the potentials in the trochoidal monochromator are adjusted to minimize the width of the electron energy distribution. However, for this work the intensity of the emitted radiation was of primary importance, and no attempt was made to produce a beam of narrow energy spread since beam currents of several microamperes were required. The unfiltered radiation from the interaction region was visible in a darkened environment and, when viewed along the optical axis, formed a sharply defined spot.

An indirectly heated oxide cathode was used for this work. This type of cathode yields sufficient emission current at a lower temperature than the directly heated filament normally used. In addition, the cathode heater is relatively easy to shield, thus reducing stray light.

After passing through the cell containing the target gas, the electrons enter a beam-collection region. The final plate, used as the electron collecting electrode, is opened at its center to permit the light produced in the collision region to be viewed. As shown in Fig. 1, the electrons are moved off the optical axis, as in the electron-beam source, and collected. In practice, it was found that the radiation intensity was enhanced by maintaining the potentials in the beam collector at that of the collision region. The gas density in the collection region was high enough to extend, effectively, the electron scattering path length. Furthermore, this configuration avoids additional broadening of the electron energy distribution by the potentials applied to efficiently collect the electron beam. Such a biasing scheme does not allow the electron-beam current to be measured accurately; however, the precise value of the current was not required as long as it remained stable.

The electron gun is housed in a stainless-steel vacuum chamber pumped by a freon-trapped oil diffusion pump. Base pressures were in the high $10^{-8}$ Torr range. The collision region is at the center of Helmholtz coils mounted external to the vacuum envelope. Direct measurements of the magnetic field strength and direction were made just outside the collision region with the electron gun in place and along the beam path with the gun removed. Variations in the field strength were $\sim 1 \%$ along the length of the collision region. The values quoted in this paper were measured near the center of the collision region with the gun removed.

The configuration of the experiment is shown schematically in Fig. 2. The focal point of the input lens $(24 \mathrm{~cm}$ focal length) was near the center of the collision region. A narrow-band interference filter, of center wavelength $5900 \AA$, selected the radiation from the $3{ }^{3} \mathrm{D} \rightarrow 2{ }^{3} \mathrm{P}$ transition. A mica 5876- $\AA$ quarter-wave plate and Polacoat 105 linear polarizer were used to analyze the circular polarization. ${ }^{13}$ The optical axes of the wave plate were located using a modification of a birefringence technique. ${ }^{14}$ As the helicity of the circular polarization is significant in this experiment, care was taken in the determination of the fast axis. For this purpose, light of known helicity was created and analyzed. The errors in the position of the fast axis and the linear polarizer are estimated to be less than 5'. A Thorn EMI 9789QA photomultiplier, operated in the discrete counting mode, measured the radiation intensity. The face of the tube was approximately $45 \mathrm{~cm}$ from the center of the Helmholtz coils. The magnetic field strength at that position was $10 \mathrm{G}$ at the strongest fields used. The tube was therefore magnetically shielded to insure that its gain was independent of changes in the magnetic field.

The control and data acquisition system, shown in Fig. 3, sets the linear polarizer, samples the radiation in-

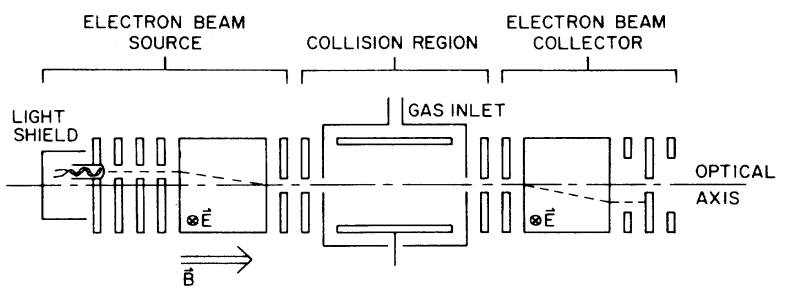

FIG. 1. Schematic of the electron-impact light source. The electron trajectory is shown by the dashed line.

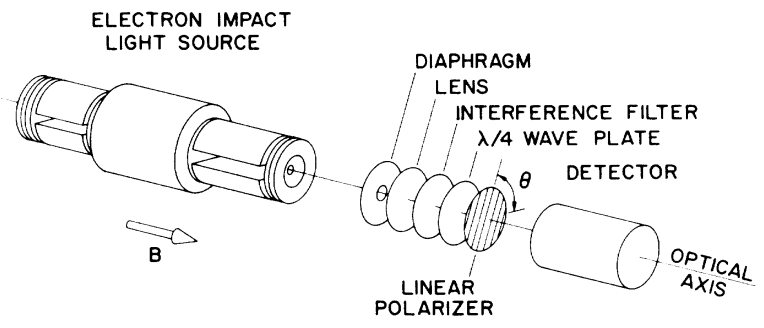

FIG. 2. Schematic arrangement of the experiment. 


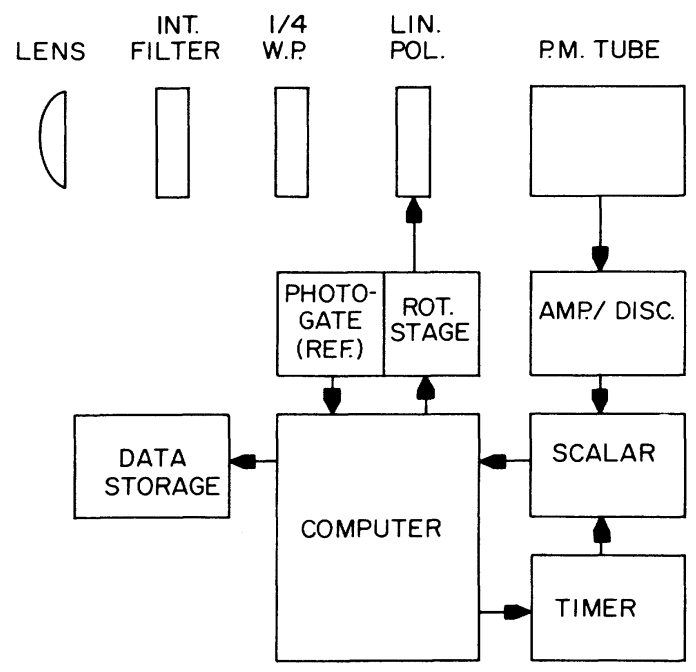

FIG. 3. Schematic of the control and data acquisition systems.

tensity, and advances the polarizer by $12^{\circ}$ through a complete revolution. These data are least-squares fit to an equation of the form

$$
I=I_{U}+I_{\mathrm{CP}} \cos ^{2}(\theta-\phi)
$$

where $I$ is the total intensity, and $I_{U}$ and $I_{\mathrm{CP}}$ are the unpolarized and circularly polarized intensities, respectively. The angular displacement of the linear polarizer $\theta$ is referenced to the fast axis of the quarter-wave plate. The parameters $I_{U}, I_{\mathrm{CP}}$, and $\phi$ are evaluated from the fit to the data. A linearly polarized component in the radiation, due to spurious reflections in the optical system, for example, or a linear polarization bias in the photomultiplier tube, would result in values of $\phi$ differing from $45^{\circ}$, the value expected for right circularly polarized radiation. However, the phase angles determined from this initial fit approximated a normal distribution centered at $43^{\circ}$ with standard deviation at $11^{\circ}$, consistent with a negligible linear-polarization bias. A final fitting to the data, with $\phi$ fixed at $45^{\circ}$, was done to reduce the variance in the estimates of $I_{U}$ and $I_{\mathrm{CP}}$. The circularpolarization fractions discussed below were calculated using the values of $I_{U}$ and $I_{\mathrm{CP}}$ determined in this manner.

A least-squares fitting routine, LMDER $1,{ }^{15}$ was used to fit the data. The routine was checked to verify that it was well behaved over the range of experimental values. For this purpose, synthetic data of the correct functional form were generated with normally distributed noise superimposed on them. Histograms of the resulting fitted input parameters were indeed centered about the input value, and the width of the distributions were well represented by the free parameter error, calculated by inversion of the curvature matrix. ${ }^{16}$

\section{RESULTS AND DISCUSSION}

The circular-polarization fraction $C$ defined as

$$
C=\frac{I_{\mathrm{RCP}}-I_{\mathrm{LCP}}}{I_{\mathrm{RCP}}+I_{\mathrm{LCP}}},
$$

where $I_{\mathrm{RCP}}$ and $I_{\mathrm{LCP}}$ are the intensities of right and left circularly polarized radiation, respectively, is the basis for comparison between results from theory and the present work. In terms of the parameters determined from the fit to Eq. (1), the magnitude of $C$ is given by

$$
C=\frac{I_{\mathrm{CP}}}{2\left(I_{U}-D\right)+I_{\mathrm{CP}}},
$$

where $D$ is the photomultiplier dark count. The sign of $C$ is determined from the value of the phase angle $\phi$, with $C>0$ for $\phi$ near $45^{\circ}$ and $C<0$ for $\phi$ near $135^{\circ}$. The linear polarizer extinction ratio was 1:340 and no correction to the measured polarization fraction was made.

Measurements of the circular-polarization fraction were obtained at 90,120,175, 260, and $350 \mathrm{G}$. All measurements were taken at an impact energy of approximately $35 \mathrm{eV}$, near the peak in the $3{ }^{3} \mathrm{D}$ excitation function, ${ }^{17}$ where the linear-polarization fraction and hence the initial alignment vary slowly with electron-impact energy. ${ }^{18}$ The gas density in the target region was estimated from the electron-beam attenuation as well as from the positive-ion current collected within the collision chamber, and our studies were carried out at three pressures, 50, 10, and $5 \mathrm{~m}$ Torr. However, as a portion of the light intensity was derived from excitation within the region of the beam collector where the gas density is less, these values can only be regarded as approximate. Precise knowledge of the gas density is not required in this experiment; although, as we discuss in more detail below, our measurements are pressure dependent and the interpretation must account for this variation.

Figure 4 shows a representative sample of the data taken with a magnetic field strength of $260 \mathrm{G}$ and collision region pressure of $50 \mathrm{mT}$ Torr. In this figure the intensity of the light propagating parallel to the magnetic

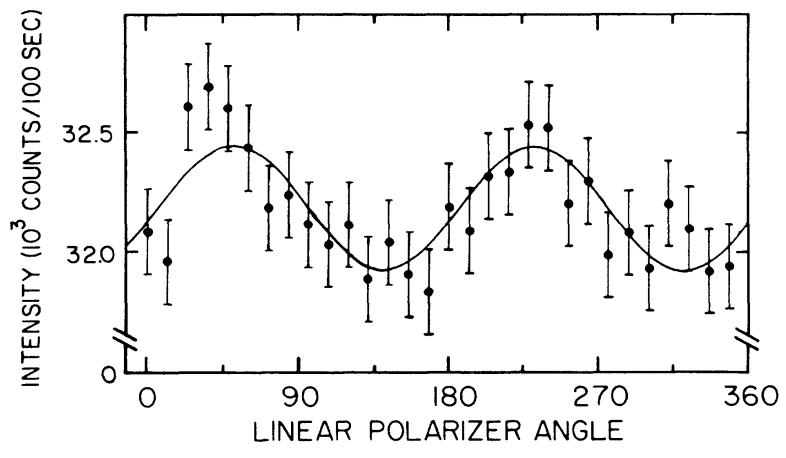

FIG. 4. Representative data taken at $260 \mathrm{G}$ and 50 mTorr. The intensity of light propagating parallel to the magnetic field direction is plotted as a function of the linear polarizer angle (degrees). The solid line is the least-squares fit to the data. 
field is plotted as a function of the linear polarizer angle. The error bars at each data point designate a $68 \%$ confidence interval. The solid curve is the least-squares fit to the data. The circular-polarization fraction determined from the fit is $0.8 \pm 0.2 \%$ and the phase shift is $51^{\circ} \pm 11^{\circ}$.

The geometry of the apparatus allowed an important consistency check on the experiment to be carried out. The magnetic field direction could be reversed without alteration of the other experimental parameters. Such a reversal, which is equivalent to repositioning the photodetector to observe light propagating antiparallel to the magnetic field, is expected to change the helicity of the observed light. This follows from the transformational properties of $O_{0}$ as discussed by Fano and Macek. ${ }^{4} \mathrm{~A}$ demonstration of this effect is shown in Fig. 5 where the curves are fit to data taken observing the radiation propagating parallel (solid line) and antiparallel (dashed line) to the magnetic field. These data were taken sequentially with all other experimental parameters unchanged. The estimated polarization fractions and phase shifts are $0.8 \pm 0.2 \%$ and $47^{\circ} \pm 11^{\circ}$, and $-0.8 \pm 0.2 \%$ and $129^{\circ} \pm 11^{\circ}$, respectively. Many sets of such data were taken with similar agreement in the magnitude of the polarization fraction. The distribution of phase angles were centered about $43^{\circ}$ and $139^{\circ}$, respectively, each with standard deviations of approximately $11^{\circ}$. The agreement with the expected $90^{\circ}$ phase shift is within the estimated error in $\phi$, and these data are consistent with a change only in the helicity upon reversal of the magnetic field.

The dependence of the measured circular-polarization fraction on the magnetic field strength is plotted in Figs. 6(a) and 6(b). The results obtained with a collision chamber pressure of $50 \mathrm{~m}$ Torr are shown in Fig. 6(a) and those obtained with pressures of 10 and $5 \mathrm{mT}$ Torr are shown in 6(b). Each point is determined from a weighted average of many sets of data taken under similar experimental conditions. The error bars shown are estimated from the scatter in the calculated polarization fractions, and thus include experimental as well as statistical uncertainties. In each case, circular-polarization

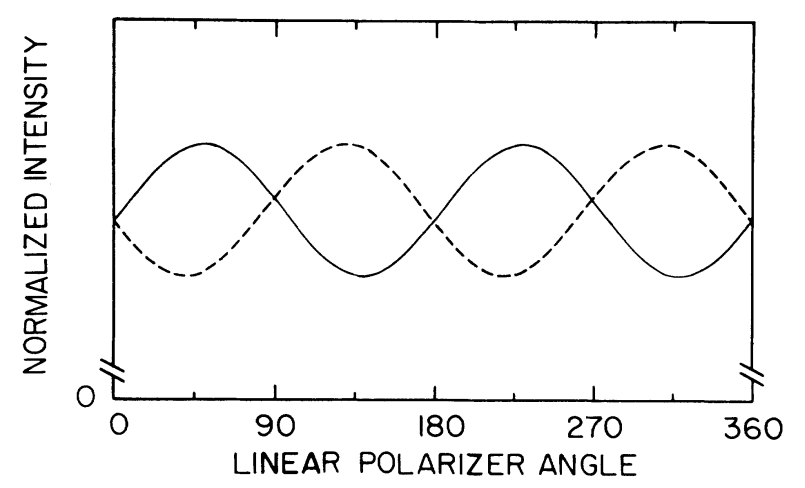

FIG. 5. Magnetic field reversal. The curves are leastsquares fits to data for light propagating parallel (solid line) and antiparallel (dashed line) to the magnetic field direction. These fits are consistent with a change only in the helicity of the radiation, as expected from theory. fractions of $1-2 \%$ in magnitude were measured. However, significant differences with pressure are observed in the magnetic-field-strength dependence, the circular polarization tending to a constant or slightly increasing function at low pressure. These differences in the field strength dependence are likely due to collisional excitation transfer, which is known to be significant for the $3^{3} \mathrm{D}$ state. ${ }^{19}$

The apparent $3{ }^{3} \mathrm{D}$ excitation function is highly pressure dependent above $5.8 \mathrm{~m}$ Torr. ${ }^{20}$ Such a function includes not only direct electron-impact excitation and cascading from higher states but also contributions from
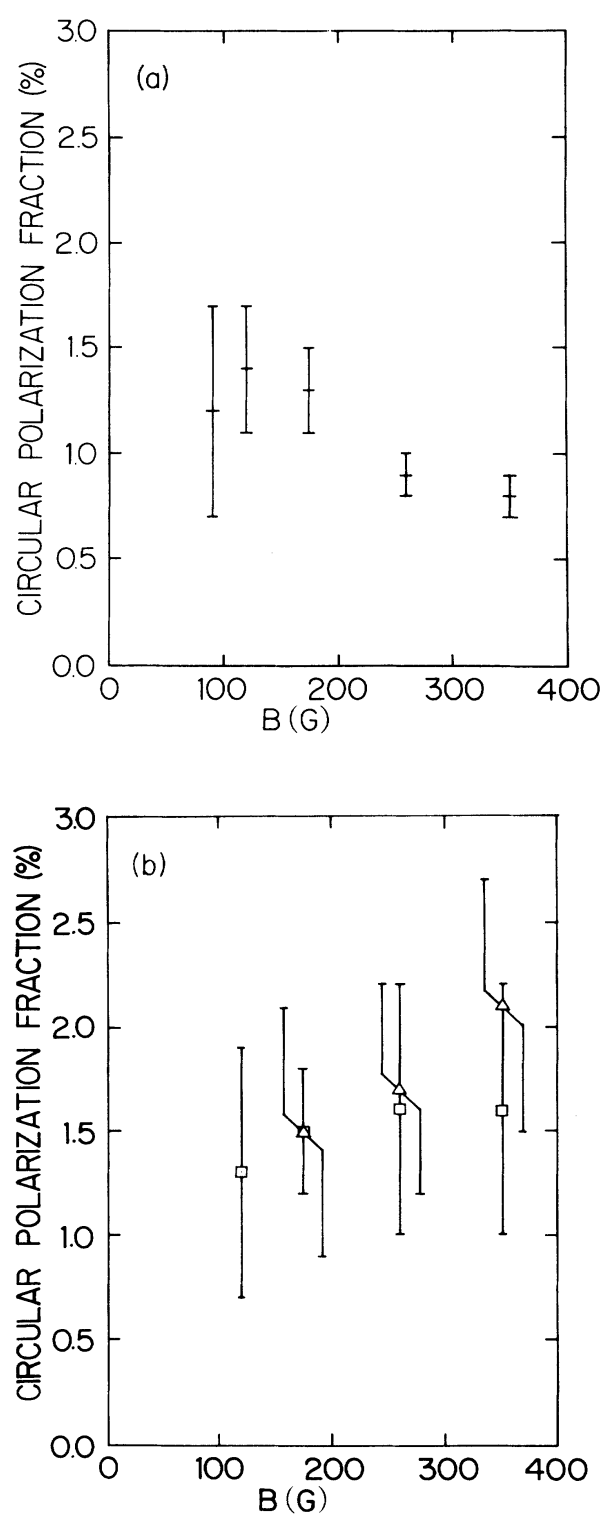

FIG. 6. Magnitude of the circular-polarization fraction as a function of magnetic field strength. The pressure in the collision region is estimated to be $50 \mathrm{mTorr}$ for the data shown in (a), while the pressures for the data shown in (b) are $10 \mathrm{mTorr}$, $(\square)$; and 5 mTorr, $(\triangle)$. 
secondary processes such as collisional transfer of excitation. Our measurements of the apparent excitation function from threshold to approximately $50 \mathrm{eV}$ were compared with those reported by St. John and Nee. ${ }^{20}$ At our highest pressure, $50 \mathrm{mTorr}$, the excitation function was in good agreement with those reported for pressures of $63 \mathrm{~m}$ Torr and higher, indicating clearly that transfer of excitation was occurring. At our intermediate pressure, the excitation function peaked near $38 \mathrm{eV}$, then decreased monotonically, characteristic of direct and cascade excitation alone.

We turn now to a discussion of the results from theory and their comparison with our measurements. Fano and Macek $^{4}$ derive an expression for dipole radiation intensity which may be used to express the experimentally determined parameters in terms of the orientation and alignment of the source. The linear-polarization fraction $P$ defined as $P=\left(I_{\|}-I_{\perp}\right) /\left(I_{\|}+I_{\perp}\right)$, measured perpendicular to the excitation axis, and the circular polarization $C$, measured parallel to the excitation axis, are pertinent to this discussion and, in their formulation, are given by

$$
P=\frac{3 h^{2}\left(l_{i}, l_{f}\right)\left\langle A_{0}\right\rangle}{\left[4+h^{2}\left(l_{i}, l_{f}\right)\left\langle A_{0}\right\rangle\right]}
$$

and

$$
C=\frac{3 h^{1}\left(l_{i}, l_{f}\right)\left\langle O_{0}\right\rangle}{\left[2-h^{2}\left(l_{i}, l_{f}\right)\left\langle A_{0}\right\rangle\right]}
$$

in the collision frame. The $h^{k}\left(l_{i}, l_{f}\right)$ are ratios of $6-j$ coefficients and are functions of $k$, the tensor order, and $l_{i}$ and $l_{f}$, the initial and final angular momenta. Alignment and orientation, if treated as explicit functions of time, describe the time evolution of the excited state. It is important to note that since $P$ and $C$ are determined from time-averaged radiation intensity measurements, $\left\langle A_{0}\right\rangle$ and $\left\langle O_{0}\right\rangle$ refer to time-averaged quantities. In the sudden-impact approximation, the time-averaged alignment and orientation are calculated as initial value problems. For the $\mathrm{He} 3{ }^{3} \mathrm{D}$ state Nehring ${ }^{2}$ calculates that

$$
\left\langle A_{0}\right\rangle=0.473 A_{0}(t=0),
$$

where $A_{0}(t=0)$ is the initial alignment, and the multiplicative constant is determined from a weighted average over the lifetime of the excited state. In this case, the excited state evolves solely due to the spin-orbit interaction. The conversion to orientation in the presence of the magnetic field is treated similarly and Nehring gives

$$
\left\langle O_{0}\right\rangle=\left[\left(\frac{5}{3(2 l+3)(2 l-1)}\right]^{1 / 2} F_{0}^{12}\right] A_{0}(t=0) \text {, }
$$

where $l$ is the angular momentum of the excited state. The time-averaged conversion coefficient $F_{0}^{12}$ is a function of the magnetic field strength and the dependence ${ }^{2}$ for the $3{ }^{3} D$ state is shown in Fig. 7 .

The results above may be used to express the circular-polarization fraction in terms of the timeaveraged alignment, determined from experimental measurement of the linear-polarization fraction $P$ and the

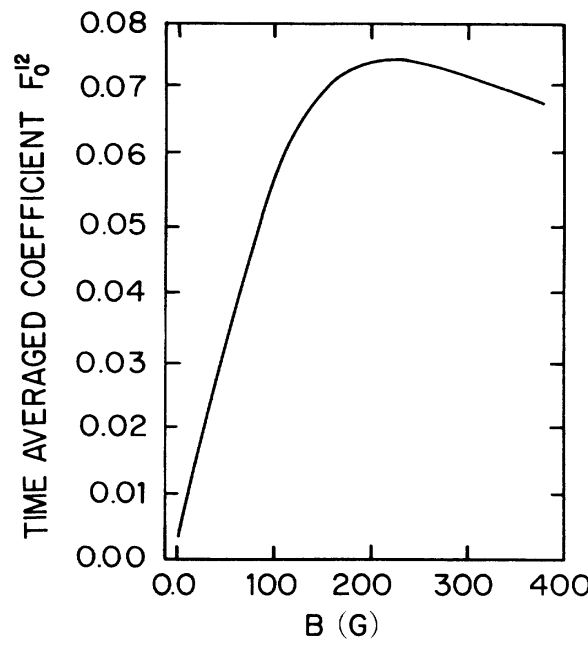

FIG. 7. Magnetic field strength dependence of the timeaveraged alignment to orientation coefficient $F_{0}^{12}$, calculated by Nehring.

calculated conversion coefficient. Linear-polarization fractions of $10 \%$ and $8 \%$ have been reported for electron-impact excitation at $35 \mathrm{eV} .^{18,21}$ These values and the results from theory predict the magnitudes of the circular-polarization fraction to be $3.2 \%$ and $2.4 \%$, respectively, at $220 \mathrm{G}$, which are in general agreement with the results from the present experimental study. The sign of the predicted polarization fraction, however, is not in agreement with that determined in the present study. This difference may be traceable to the sign of the calculated conversion coefficient, which was not central to Nehring's study and may not have been consistently treated. ${ }^{22}$

We now consider the magnetic field dependence of the polarization fraction. Because the circular-polarization fraction is proportional to $F_{0}^{12}$, the function shown in Fig. 7, aside from a scaling factor, should also describe the magnetic field dependence of the observed signal as predicted by theory. It must be noted, however, that these calculations allow only for direct excitation of the $3{ }^{3} \mathrm{D}$ state. No contributions from higher excited states which cascade to the ${ }^{3} D$ state are included. These other excited states will undergo their own characteristic alignment to orientation conversion and will contribute to the observed signal in a manner that has not been accounted for in the theory.

The measured signal may also differ from that predicted by theory because of secondary excitation mechanisms such as collisional excitation transfer. At sufficiently high gas densities, collisions between ground-state atoms and excited-state atoms in the $n^{1} P$ states have been found to yield $\mathrm{He}^{*}(n F)$ with high probability. ${ }^{20,23}$ The latter may then photodecay to $\mathrm{He}^{*}$ $\left(3{ }^{3} D\right)$. At high pressures, there may be population of the $n^{1} P$ states due to radiation trapping effects, in addition to direct electron-impact excitation, since the $n^{1} P$ states are dipole allowed from the ground state. Thus a large fraction of the total intensity may be attributed to 
secondary effects. We estimate from the data reported by St. John and $\mathrm{Nee}^{20}$ that approximately $40 \%$ of the total intensity in our high pressure data is due to secondary excitation.

Turning now to our results shown in Figs. 6(a) and 6(b), the data taken at $50 \mathrm{mTorr}$ are subject to the secondary excitation mechanisms mentioned above, as shown by the shape of the $3{ }^{3} \mathrm{D}$ apparent excitation function. The two sets of measurements at lower pressures, on the other hand, appear to be free of these effects and thus display the magnetic field dependence of the direct and cascade excitation alone. Unfortunately, neither of the sets of measurements can therefore be directly compared to that derived by Nehring. Nevertheless, the trends in the curves appear to be consistent with the appropriate excitation mechanism.

The initial alignment and orientation of the $\mathrm{He} 3{ }^{3} \mathrm{D}$ state depends on the excitation mechanism. We argue that two mechanisms must be considered to interpret our results: the secondary excitation sequence described above and direct electron-impact excitation. Secondary excitation requires collisional transfer of excitation, and since the He velocity distribution is essentially isotropic, the $3{ }^{3} \mathrm{D}$ excited-state population that is a consequence of this mechanism is created with reduced alignment and orientation. We will assume for purposes of this discussion that this mechanism yields essentially unaligned and unoriented $3{ }^{3} \mathrm{D}$ states. The net effect of the secondary excitation is the addition of an unpolarized component to the radiation. This interpretation is corroborated by experimental evidence of a reduced linear-polarization fraction, and hence reduced initial alignment, when secondary excitation processes are significant. ${ }^{18,21}$ Consistent with this, in our high-pressure data the circularpolarization fraction is systematically smaller than in the lower-pressure measurements. To estimate the size of the change in the circular-polarization fraction expected from secondary excitation, the component of net circular polarization, which is a small fraction of the total intensity, is assumed constant and an unpolarized component equal to $40 \%$ of the initial intensity (as estimated above from St. John and Nee) is added to the total intensity. These assumptions yield a circular-polarization fraction reduced by $\sim 30 \%$, in reasonable agreement with that observed at $350 \mathrm{G}$ in Fig. 6.

Gas collisions have the additional effect of destroying the direct cascade sequence to the $3^{3} \mathrm{D}$ state and thus reducing alignment and orientation which may be transferred to the $3{ }^{3} \mathrm{D}$ state by cascade from states excited by electron impact. Where secondary excitation is dominant, the residual circular polarization would arise predominantly from $3{ }^{3} \mathrm{D}$ states directly excited by electron impact, and the magnetic field dependence would approach that for direct electron-impact excitation. Thus the decline in the signal as the magnetic field increases, shown in Fig. 6(a), appears to be consistent with that predicted by Nehring. At low pressure, the signal tends toward a constant value or rises slightly with increasing magnetic field. We argue that this reflects the contributions from the cascade states.

In conclusion, this work demonstrates the alignment to orientation conversion mechanism postulated by Kemp, Macek, and Nehring. The measured circularpolarization fractions are of the order expected from the calculations and independent measurements of the linear-polarization fraction. Furthermore, the magnetic field dependence at high pressure agrees qualitatively with that of Nehring where, we argue, the field strength dependence approaches that expected from directly excited $\mathrm{He} 3{ }^{3} \mathrm{D}$. At low pressure, the cascade from other directly excited states must be properly included to obtain quantitative agreement with experiment. The effects of the magnetic field on the time evolution of each excited state must also be included for comparison with the present case. The excitation functions taken in the present work indicate that the intermediate- and lowpressure data were taken under conditions which approach the low-pressure limit. Our results may therefore be useful for comparison with a more complete theoretical treatment.

Kemp et al. ${ }^{1}$ have suggested that the effect described here may account for the anomalous circular polarization observed in sunspots, and that it may be used to obtain information on the local magnetic field strengths within sunspots. We suggest that there may also be application as a plasma diagnostic.

\section{ACKNOWLEDGMENTS}

This work was supported by the National Science Foundation, Grant Nos. CPE-8411560 and PHY8401328. We wish to thank J. H. Macek for suggesting this problem and for his many insightful comments on this work. We also thank D. H. Jaecks and D. B. Billesbach for the loan of optical components and D. W. Schlitt for assistance with the data analysis software.
${ }^{1}$ J. C. Kemp, J. H. Macek, and F. W. Nehring, Astrophys. J. 278, 863 (1984).

${ }^{2}$ F. W. Nehring, Ph.D. thesis, University of Nebraska-Lincoln, 1979.

${ }^{3}$ K. Blum, Density Matrix Theory and Applications (Plenum, New York, 1981).

${ }^{4}$ U. Fano and J. H. Macek, Rev. Mod. Phys. 45, 553 (1973).

${ }^{5}$ M. Lombardi, in Beam-foil Spectroscopy, edited by I. A. Sellin and D. J. Pegg (Plenum, New York, 1975), Vol. 2.

${ }^{6}$ D. E. Rees, Proc.—Astron. Soc. Aust. 4, 335 (1982).
${ }^{7}$ J. C. Lehmann, Phys. Rev. 178, 153 (1969).

${ }^{8}$ L. Sanche and G. J. Schulz, Phys. Rev. A 5, 1672 (1972).

${ }^{9}$ A. Stamatovic and G. J. Schulz, Rev. Sci. Instrum. 41, 423 (1970).

${ }^{10}$ D. Roy, Rev. Sci. Instrum. 43, 535 (1972).

${ }^{11}$ M. R. McMillan and J. H. Moore, Rev. Sci. Instrum. 51, 944 (1980).

${ }^{12}$ A. R. Johnston and P. D. Burrow, J. Electron Spectrosc. Relat. Phenom. 25, 119 (1982).

${ }^{13}$ D. Clarke and J. F. Grainger, Polarized Light and Optical 
Measurement (Pergamon, Oxford, 1971).

${ }^{14}$ I. G. Wood and A. M. Glaszer, J. Appl. Crystallogr. 13, 217 (1980).

${ }^{15}$ B. S. Garbow, K. E. Hillstrom, and J. J. More, Argonne National Laboratory, Report No. ANL-80-74, Minpack Project, March 1980 (unpublished).

${ }^{16} \mathrm{P}$. R. Bevington, Data Reduction and Error Analysis for the Physical Sciences (McGraw-Hill, New York, 1969).

${ }^{17}$ R. M. St. John, F. L. Miller, and C. C. Lin, Phys. Rev. 134, A888 (1964).
${ }^{18}$ R. H. McFarland and E. A. Soltysik, Phys. Rev. 127, 2090 (1962).

${ }^{19}$ R. M. St. John and R. G. Fowler, Phys. Rev. 122, 1813 (1961).

${ }^{20}$ R. M. St. John and T. W. Nee, J. Opt. Soc. Am. 55, 426 (1965).

${ }^{21}$ I. P. Bogdanova and M. P. Chaika, Opt. Spektrosk. 47, 22 (1979) [Opt. Spectrosc. (USSR) 47, 22 (1979)].

${ }^{22}$ F. W. Nehring (private communication).

${ }^{23}$ C. C. Lin and R. G. Fowler, Ann. Phys. (NY) 15, 461 (1961). 